\title{
Le défi thérapeutique dans la région antérieure maxillaire : à propos d'un cas
}

\section{The therapeutical challenge in maxillar area: about a case report}

\section{MOTS-CLEFS :}

- Esthétique, guide antérieur, chirurgie parodontale corrective, axiographie

\section{KEYWORDS:}

- Aesthetic, anterior guidance, corrective periodontal surgery, axiography

\section{Résumé}

Dans la gestion de l'édentement antérieur, l'esthétique est l'un des paramètres essentiels. Il dépend de l'étendue de l'édentement, de la valeur de la crête alvéolaire du maxillaire supérieur ainsi que de la qualité du guide antérieur. Mais la recherche de l'esthétique dento-labiale a surtout une dimension humaine qui dépasse le cadre des techniques opératoires. Le praticien fait donc face ò un double défi : - recopier la nature pour créer l'illusion du naturel en denture artificielle, tout en respectant les différents paramètres biologiques (le guide antérieur, la santé parodontale...);

- répondre à la demande immédiate du patient qui obéit au canon socio-culturel occidental de dents jeunes, claires, petites et bien rangées.Face à ce défi, la prise en compte d'une chronologie raisonnée de réalisation du plan de traitement s'avère judicieuse pour faciliter les étapes thérapeutiques et diminuer la temporisation. Nous allons exposer, à travers un cas clinique, les différentes étapes (parodontales et prothétiques) de la gestion d'une réhabilitation prothétique antérieure.

\section{Abstract}

When managing an anterior teeth mess, aesthetics is one of the basic parameters to work on.

It depends on the extent of teeth loss, the value of the alveolar ridge of the maxilla and the quality of anterior guidance. However, the seek of dento-labial aesthetic has a human dimension which is beyond the scope of surgical techniques.

The practitioner therefore has a dual challenge:

- creating the illusion on artificial teeth by copying on nature, while respecting the different biological parameters (the previous guide, periodontal health...);

- responding to the immediate request of the patient who obeys the socio-cultural canon of young, small, and on-a-line lighter teeth. To face this challenge, a rational timeline for carrying out the treatment plan should be taken into consideration in order to facilitate therapeutic steps and reduce the delay. In this work, we will present, through a clinical case, the different steps (periodontal and prosthetic) of anterior prosthetic rehabilitation.
AOS 2014;270:24-30 DOI: $10.1051 /$ aos/2014405 (C) EDP Sciences 2014

Rachid EL OUALI, Professeur agrégé de l'enseignement supérieur,
Service de prothèse fixée du CCTD, CHU Ibn Sina, Rabat,
Faculté de Médecine dentaire, Université Mohamed V
Loubna EL FIGUIGUI, Médecin Résident, PHDsService de prothèse fixée du
CCTD, CHU Ibn Sina, Rabat, faculté de Médecine dentaire, Université Mohamed V
Ismaili ZOUHAIR, Professeur de l'enseignement supérieur,
Service de parodontologie du CCTD, CHU Ibn Sina, Rabat
Amal EL YAMANI, Professeur de l'enseignement supérieur et chef du service
de prothèse fixée du CCTD, CHU Ibn Sina, Rabat




\section{INTRODUCTION}

Dans la gestion de lédentement antérieur, l'esthétique est l'un des paramètres essentiels. Il dépend de la forme, de la teinte, de la visibilité du rempart alvéolaire du maxillaire supérieur, mais aussi de la position de la lèvre supérieure par rapport aux différents repères esthétiques.

Le fait de s'intéresser à l'esthétique des reconstructions antérieures ne doit en aucun cas détourner l'attention du clinicien de la nécessité de restaurer un guide antérieur fonctionnel.Le guide antérieur joue un rôle important au niveau de la physiologie du système manducateur. En effet, les dents antérieures participent à la fonction statique de calage mandibulaire, à la fonction dynamique de nutrition et de phonation et à la fonction cinématique de guidage de la mandibule $[3,4]$.
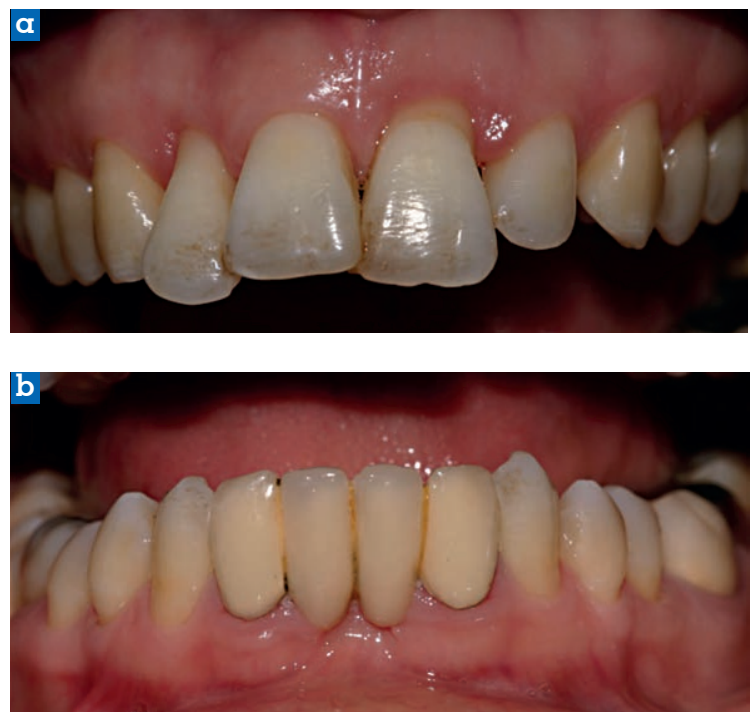

$\triangle$ Fig. 1 :

a. Les quatre incisives supérieures de la patiente présentant un chevauchement et des égressions

b. Bridge inférieur de la 32 à la 42 de morphologie approximative avec manque d'étanchéité cervicale.
Elles protègent les structures dentaires, parodontales, musculaires et articulaires grâce à la prise en charge des mouvements mandibulaires. Cette prise en charge se fait grâce à l'anatomie particulière de ce groupe de dents, mais aussi et surtout grâce à une composante proprioceptive, responsable des phénomènes d'adaptation et de régulation des cycles masticatoires.

\section{PRÉSENTATION DU CAS}

Madame M., âgée de quarante ans, exempte de pathologie générale, s’est présentée pour des soucis d'ordre esthétique et fonctionnel. La patiente souffre d'une gêne fonctionnelle suite à des mobilités dentaires consécutives à un traitement orthodontique de longue durée. La demande esthétique reste par ailleurs forte. L'examen dentaire (fig. 1a et $\mathbf{1 b}$ ) montre au niveau du bloc incisif supérieur des malpositions ainsi que de légères dysplasies de lémail. Au niveau du bloc incisif inférieur, un bridge remplaçant la 31 et la 41, de morphologie approximative, nécessiterait, par ailleurs, une restauration.

Sur le plan parodontal, on note au niveau des quatre incisives supérieures une mobilité de 2 à 3 degrés, des poches qui varient de 6 à $8 \mathrm{~mm}$ et des récessions de $1 \mathrm{~mm}$ à $3 \mathrm{~mm}$, notamment au niveau de la 21,12 , $13,23$.

L'examen radiographique (fig. 2) révèle une lyse osseuse atteignant le tiers apical au niveau de la $11,12,22,23$, des résorptions apicales importantes aux niveaux des quatre incisives supérieures et des traitements endodontiques défectueux sur les 31 et 41 piliers du bridge inférieur. Sur le plan esthétique, les bords libres du groupe incisivo-canin maxillaire forment normalement une ligne harmonieuse à légère concavité supérieure, parallèle à la lèvre inférieure durant le sourire.

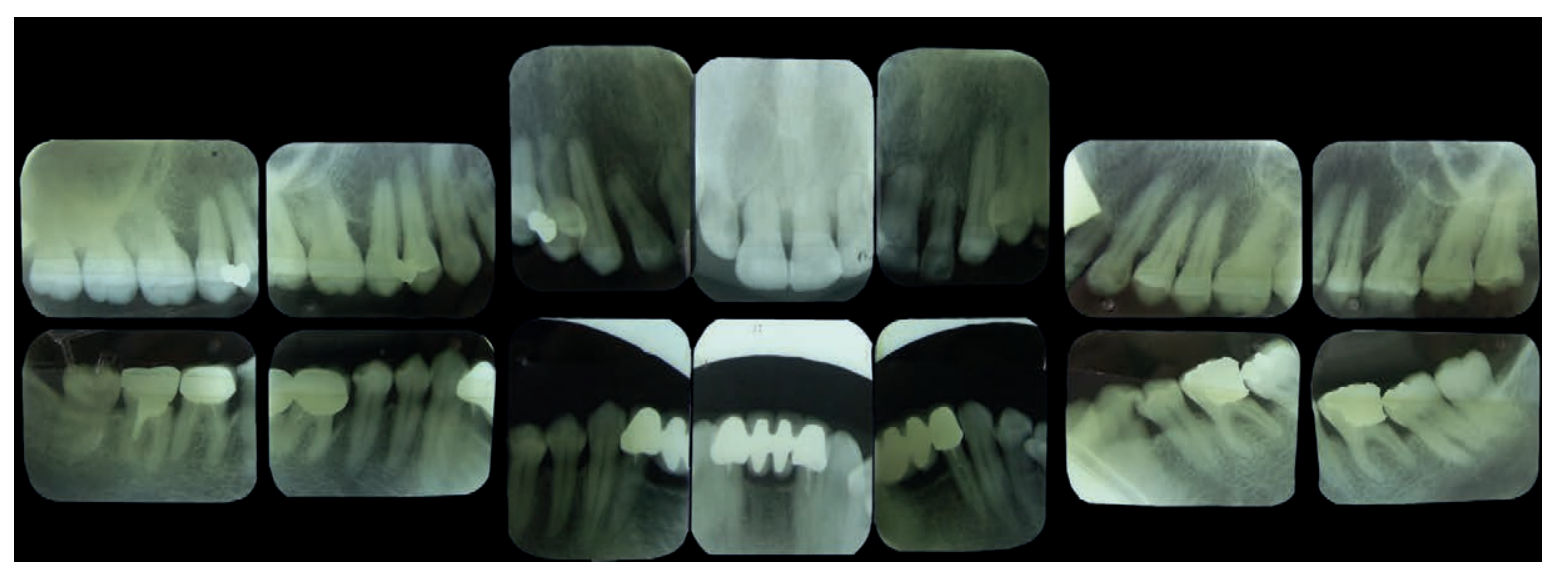

$\triangle$ Fig. 2 :

Bilan radiographique rétro alvéolaire de la situation initiale qui met en évidence la résorption osseuse et radiculaire marquée au niveau du bloc incisif supérieur. 

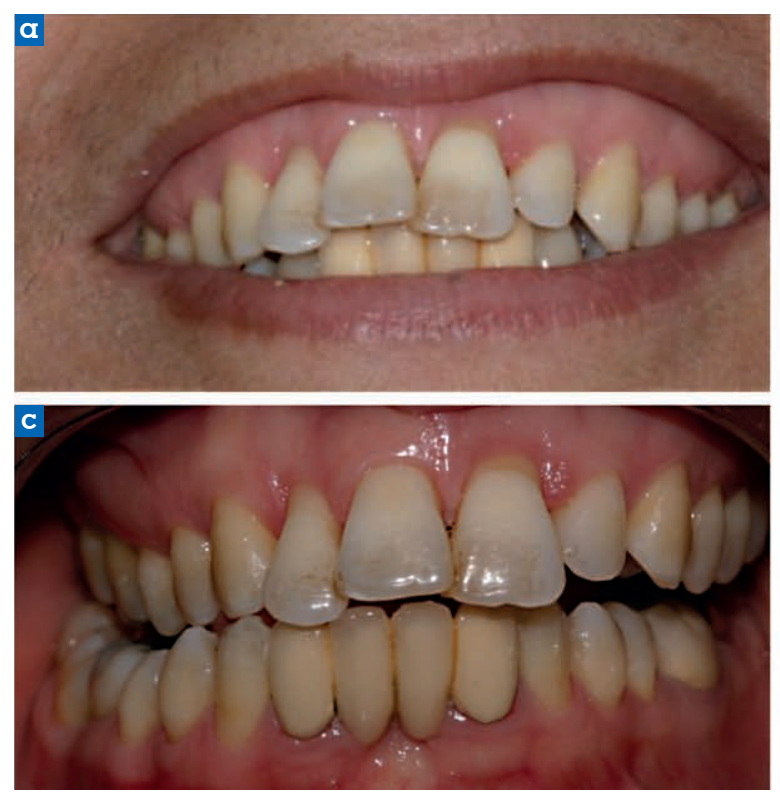

Fig. 3 :

a. Sourire gingival de la patiente avec un non alignement des collets b. Propulsion chez la patiente

c. Latéralité droite

d. Latéralité gauche.

Pour notre patiente, la ligne du sourire est sinueuse et l'alignement des collets est absent. Notons que le sourire de la patiente est gingival (fig. 3a).

Évaluation clinique du guide antérieur.

L'examen du guide antérieur passe par l'examen des contacts occlusaux : lors des excursions mandibulaires à direction horizontale (propulsion, latéralités), les surfaces palatines des dents maxillaires antérieures constituent des surfaces de glissement pour leurs antagonistes. En OIM, les contacts sont symétriques entre les bords incisifs et chaque pointe canine mandibulaire, et le point d'inflexion de la courbure palatine des crêtes proximales maxillaires.

En propulsion, le bord libre des incisives mandibulaires glisse de l'OIM selon des trajets linéaires continus, ou de relais, symétriques le long des surfaces de guidage maxillaires, jusquau bout à bout incisif, avec un désengrènement postérieur $[1,4,7,9,10]$.

En latéralité, les pointes canines mandibulaires glissent sur le pan mésial de larête médiane triangulaire de la canine maxillaire, avec désengrènement immédiat des côtés travaillants et non travaillants. C'est la fonction canine $[1,4,9]$.En cas de fonction de groupe, plusieurs dents (postérieures et/ou antérieures) adjacentes à la canine sont en contact sur une partie ou tout le trajet de déplacement, et contribuent à guider la mandibule [ 1 , 4, 9].Le guide peut être considéré comme fonctionnel et doit être conservé, s'il possède les principales caractéristiques prédéterminées. Ainsi, le guide peut être considéré comme afonctionnel lorsqu'il n'existe pas (béance par exemple), ou s'il n'assure pas une désocclusion immédiate lors de mouvement de propulsion et de
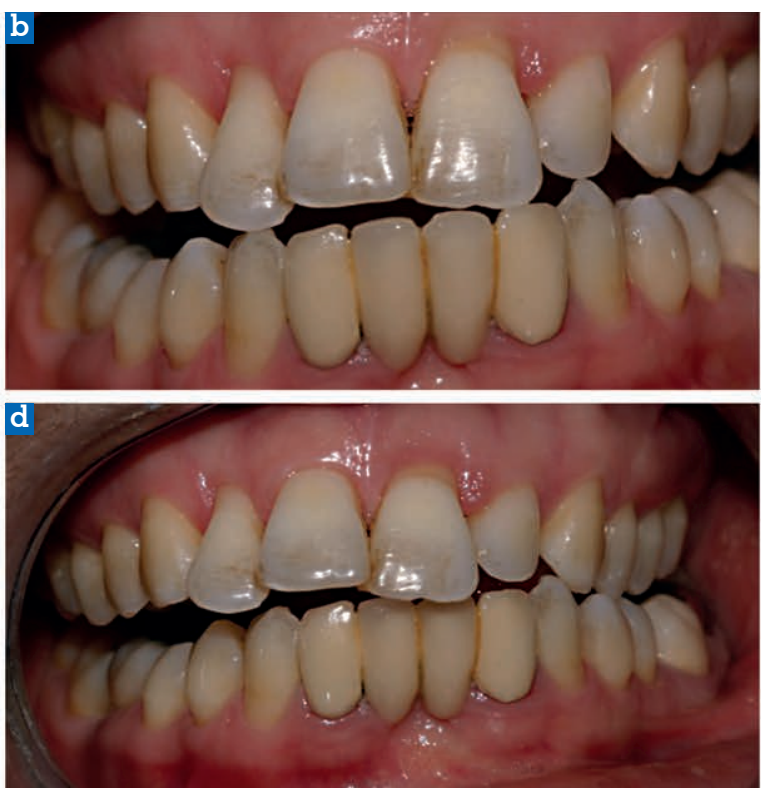

latéralisation en présence d'interférence. Le guide est dit dysfonctionnel, lorsqu'il existe mais qu'il constitue une entrave aux mouvements mandibulaires $[5,10]$. Notre patiente présente un guide antérieur afonctionnel du fait de :

Ila propulsion assurée par la 21 et la 12 avec 41,32 du bridge inférieur et la 43 , avec interférences postérieures droites (fig. $\mathbf{3 b}$ ) ;

Ila latéralité droite assurée par une fonction antérolatérale avec interférences antérieures (fig. 3c);

Ila latéralité gauche assurée par une fonction canine avec interférences antérieures (fig. 3d).

\section{DÉCISION THÉRAPEUTIQUE}

Le choix thérapeutique délaissant d'emblée pour des raisons évidentes de confort, d'esthétique et d'âge de la patiente la prothèse amovible, se discute entre un bridge conventionnel ou une restauration sur implant. La solution implantaire est refusée par la patiente et son entourage.

La solution prothétique définitive retenue a donc été le scellement d'un bridge céramo-métallique supérieur de 24-23-22-21-11-12-13-14 et un bridge inférieur céramo-métallique de 33-32-31-41-42-43.

I Pour le bridge inférieur de 33- 32-31-41-42 -43 :

I dépose de bridge 32-31-41-42 ;

I reprise de traitement endodontique de la 32 et 42 .

I Pour le bridge 24-23-22-21-11-12-13-14 :

II traitement endodontique 23-24-14-13-33-43;

I extraction 12-11-21-22; 

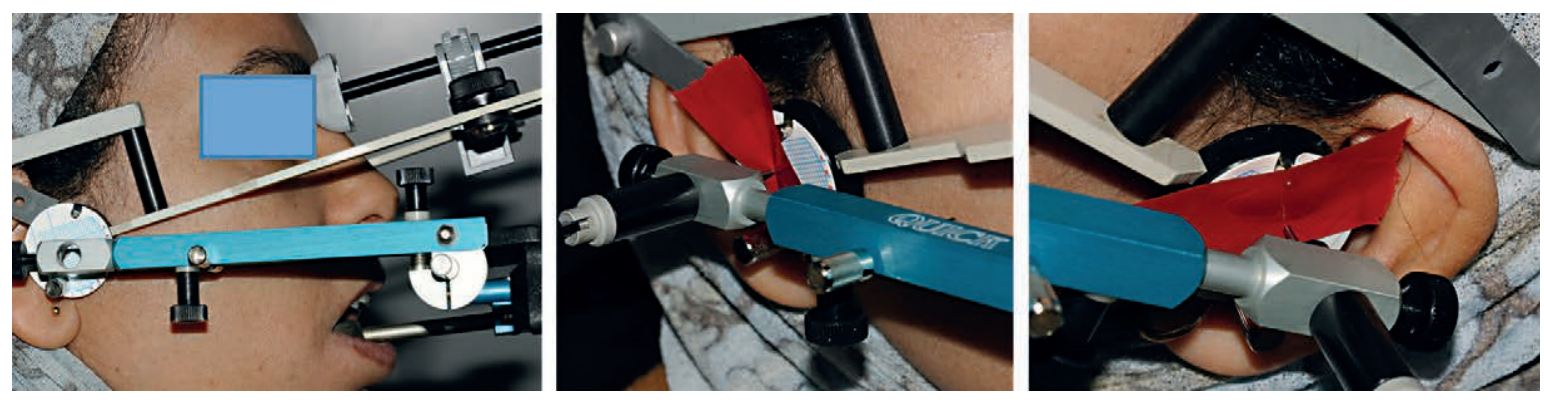

$\triangle$ Fig. 4 :

Réalisation de l'axiographie.
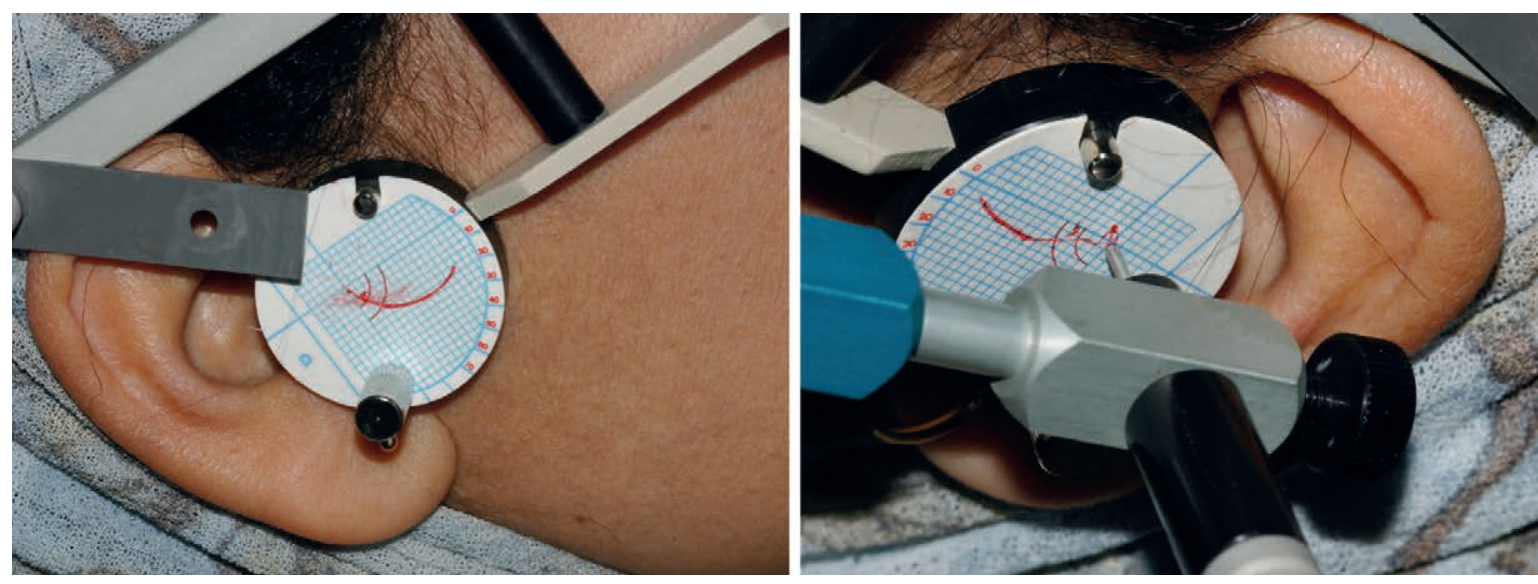

Fig. 5 :

Tracés agiographiques curvilignes et normaux chez notre patiente.

I préservation alvéolaire postextractionnelle de la crête supérieure pour réduire un effondrement en rapport avec le remodelage osseux après extractions dentaires.

\section{ÉTAPE DE RÉALISATION}

Vu que le guide antérieur est inexistant, sa confection s'impose avant la reconstruction prothétique éventuelle. Les déterminants antérieurs étant fallacieux et ne remplissant pas leur rôle salvateur, seuls les déterminants condyliens ou postérieurs sont utilisables. C’est donc à partir de leur enregistrement que le guidage antérieur sera confectionné $[4,6]$.

\section{Axiographe et programmation de l'articulateur}

Les tracés axiographiques sagittaux des mouvements de latéralité, centrifuges et centripètes, ainsi que de louverture buccale, sont repérés par rapport au plan axio-orbitaire. Ces tracés permettent d'établir un diagnostic fonctionnel des articulations temporo-mandibulaires (ATM) et de programmer les pentes condyliennes de l'articulateur.En général, le tracé de grande ouverture ou de propulsion est confondu et
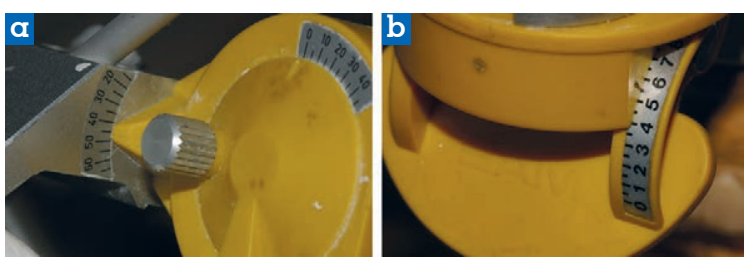

$\triangle$ Fig. 6:

a. Programmation de la pente condylienne

b. Programmation de la pente incisive en inclinant la table incisive de l'articulateur de $10^{\circ}$ par apport à la pente condylienne.

curviligne, reflétant l'anatomie du processus temporal. Ce tracé représente la pente condylienne. Elle peut être mesurée grâce à des arcs de cercle sur les drapeaux à $3 \mathrm{~mm}$ ou à $5 \mathrm{~mm}$. La corde joignant le point de départ du mouvement à son intersection avec l'arc de cercle à 3 ou $5 \mathrm{~mm}$ donne la valeur de pente condylienne à 3 ou $5 \mathrm{~mm}$ qui peut être programmée sur le boîtier condylien de l'articulateur. En général, la pente condylienne mesurée à $5 \mathrm{~mm}$ est adoptée car elle correspond au bout à bout incisif $[6,7,8]$.Pour Madame M., ces tracés ne mettent pas en évidence de pathologie des ATM. Lamplitude des mouvements est quasi normale (fig. 4 et 5). À partir de notre tracé et par des méthodes de calcul de conversion, on programme les déterminants postérieurs de notre articulateur (type SAM), on utilise une table incisive réglable que l'on incline de $10^{\circ}$ par rapport à la pente condylienne la plus pentue obtenue dans notre tracé axiographique (fig. 6a et 6b). 
Cette inclinaison dans le plan sagittal de la table incisive guide sa tige en propulsion et aussi en latéralité.Larc facial est le moyen de monter le modèle maxillaire selon le plan axio-orbitaire. Pour le mon-

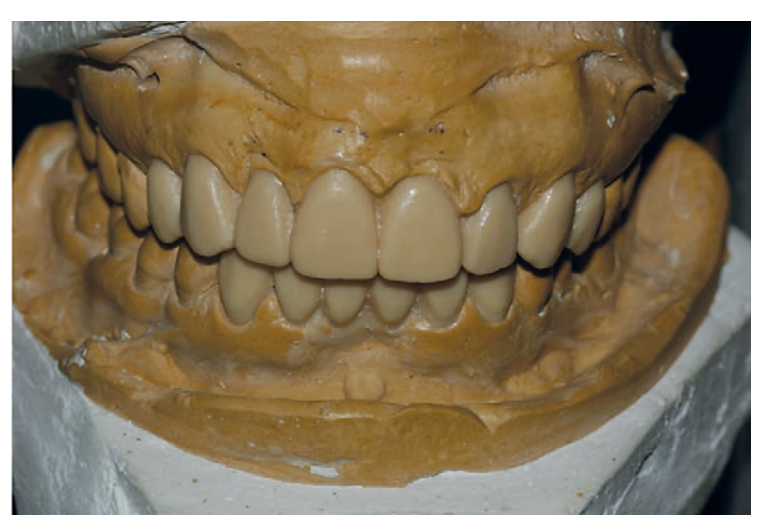

$\triangle$ Fig. 7 :

Le wax up de la patiente.

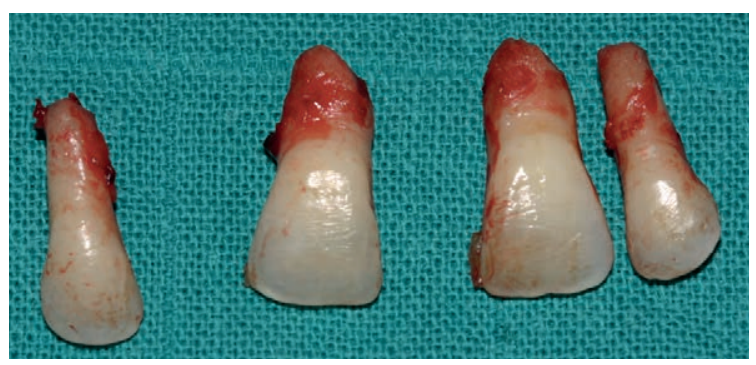

$\triangle$ Fig. 8 :

La photo montre clairement la résorption radiculaire au niveau les quatre incisives extraites.

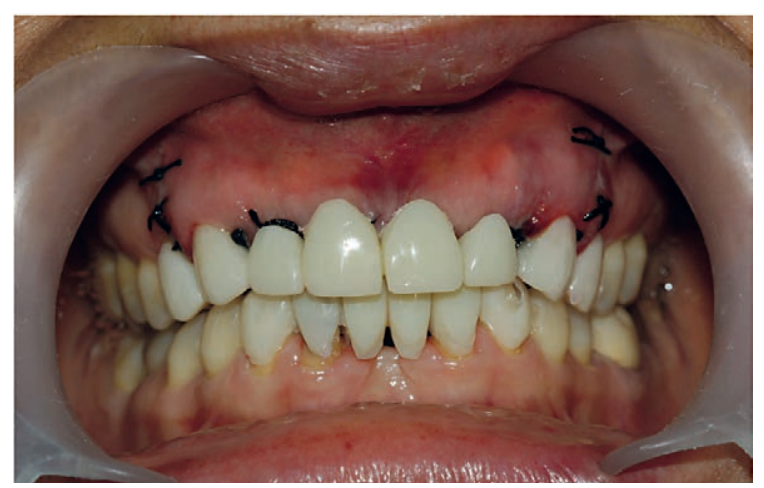

Fig. 9 :

Pose de prothèses provisoires issues du wax up immédiatement après la fin de la chirurgie pour guider la cicatrisation. tage du modèle mandibulaire, la position de référence retenue a été la relation centrée. Cette position a été recherchée par manipulation.

Larticulateur ainsi programmé antérieurement et postérieurement avec les modèles montés est envoyé au laboratoire pour confectionner les wax up et les prothèses provisoires.

\section{Réalisation des wax up, des prothèses provisoires et leur validation en bouche}

Le prothésiste confectionne les faces linguales en tenant compte des données mises à sa disposition. La restauration temporaire reproduit les contours de cire de diagnostic (fig. 7). Celles-ci étant prêtes, on procède à la préparation en bouche des dents antérieures maxillaires et on rebase les prothèses provisoires en bouche.Les faces palatines de celles-ci seront alors modelées par adjonction ou meulage de résine afin d'approcher au mieux la morphologie adaptée à un guide antérieur correct.

\section{Aménagement parodontal}

Il n'y a pas d'esthétique sans parodonte sain. Nos traitements commencent toujours par une mise en condition parodontale.

Dans notre cas, la patiente neccessite, après assainissement parodontal, une extraction des 4 incisives supérieures qui présentent une lyse osseuse importante, nécessitant ainsi une chirurgie parodontale corrective, type comblement osseux (fig. 8).

Après assainissement parodontal, les deux canines sont taillées. Immédiatement après, les quatre incisives supérieures sont extraites et un comblement des alvéoles avec de l'os d'origine bovine recouvert par une membrane non résorbable en collagène.

Un lambeau tracté coronairement permet ensuite la fermeture du site, puis la prothèse est scellée provisoirement (fig. 9).

Après 8 semaines, la membrane est dégagée et le site est refermé (fig. 10).

La cicatrisation est illustrée figure 11.
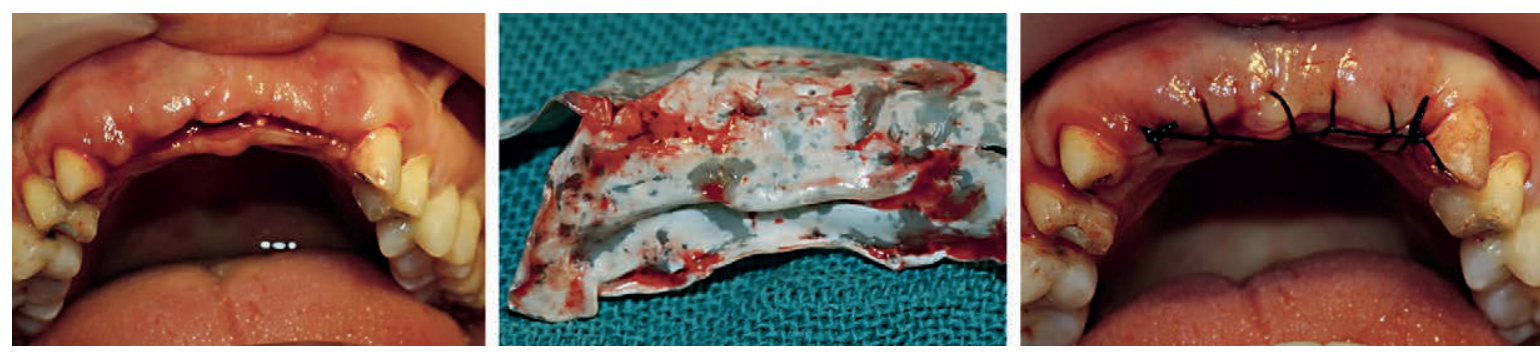

$\triangle$ Fig. 10 :

Dégagement de la membrane non résorbable après 8 semaines. 


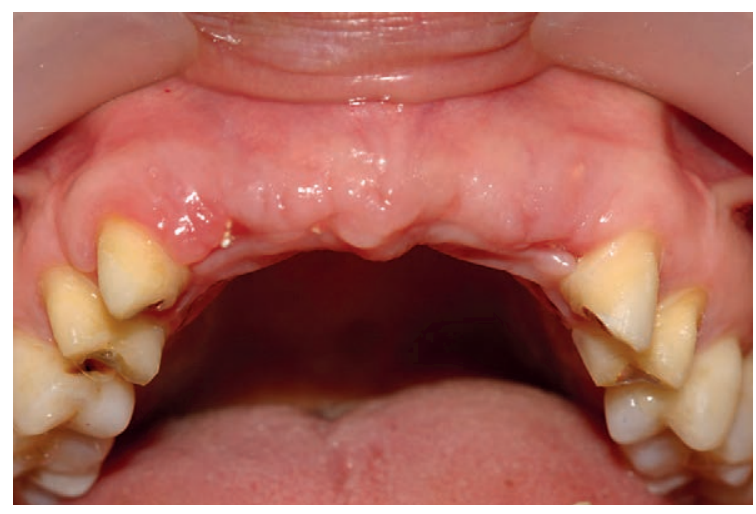

$\triangle$ Fig. 11 :

Crête après cicatrisation.
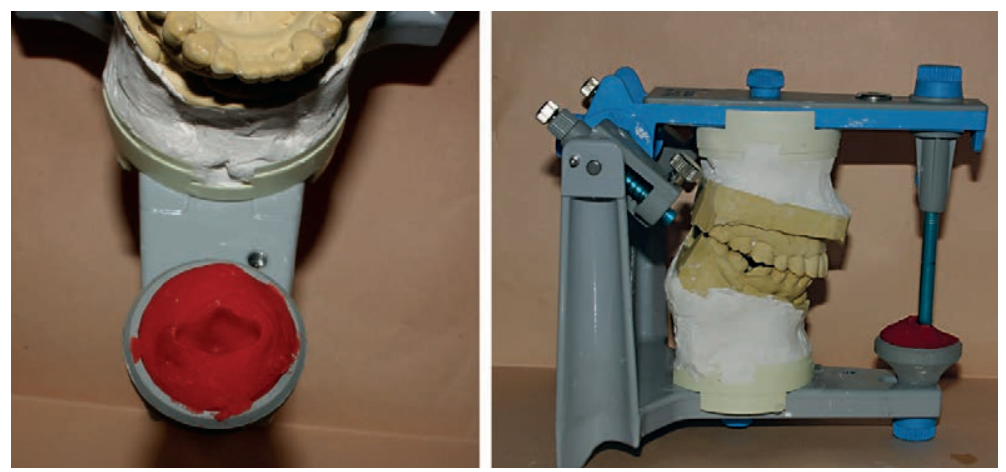

$\triangle$ Fig. 12 :

Table incisive avec l'enveloppe des mouvements tracée dans la résine qui servira à lélaboration et à léquilibration de la prothèse définitive.

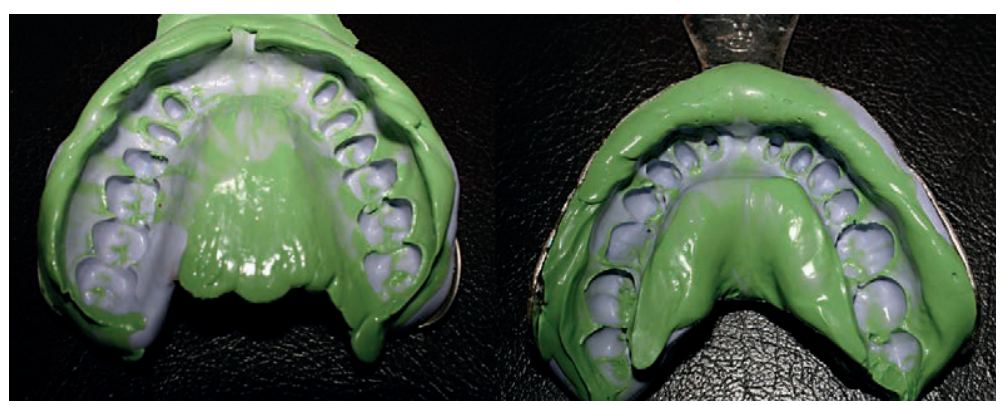

$\triangle$ Fig. 13 :

Empreinte secondaire supérieure et inférieure.

\section{Enregistrement du guide antérieur :} réalisation de la table incisive

Lorsque le guidage antérieur est parfaitement restauré en bouche à l'aide des prothèses provisoires, nous procédons alors à son enregistrement en vue de réaliser nos prothèses définitives :

Des empreintes des arcades maxillaires et mandibulaires sont prises avec les prothèses provisoires en bouche. Les modèles issus de ces empreintes sont montés sur articulateur. Le montage se fait en intercuspidation maximale. Afin que le guide antérieur soit correct, une table incisive personnalisée est confectionnée. Ce type de table est conçu pour que la branche supérieure de l'articulateur puisse effectuer les mouvements d'excursion, même lorsque le modèle mandibulaire est séparé de l'articulateur.De la résine est placée sur la table incisive. On guide les dents en propulsion, en latéralité et dans les mouvements mandibulaires. La tige incisive trace une enveloppe de mouvements dans la résine qui servira à l'élaboration et à l'équilibration de la prothèse définitive (fig. 12).

Elle sera utilisée tout le temps de la confection et de l'équilibration de la prothèse à réaliser.

\section{Enregistrement de l'occlusion statique}

Après l'enregistrement du guide antérieur, on a procédé à la prise d'empreintes des préparations en bouche et à leur montage sur articulateur (fig. 13). Les modèles issus de l'empreinte des provisoires permettent de réaliser des montages croisés.Ainsi on disposera de quatre possibilités de montage :

$\checkmark$ les modèles issus des provisoires (fig. 14a);

$\checkmark$ le modèle supérieur de travail avec le modèle des provisoires inférieures (fig. 14b) ;

$\checkmark$ le modèle inférieur de travail avec le modèle des provisoires supérieures (fig. 14c);

I les modèles de travail (fig. 14d).
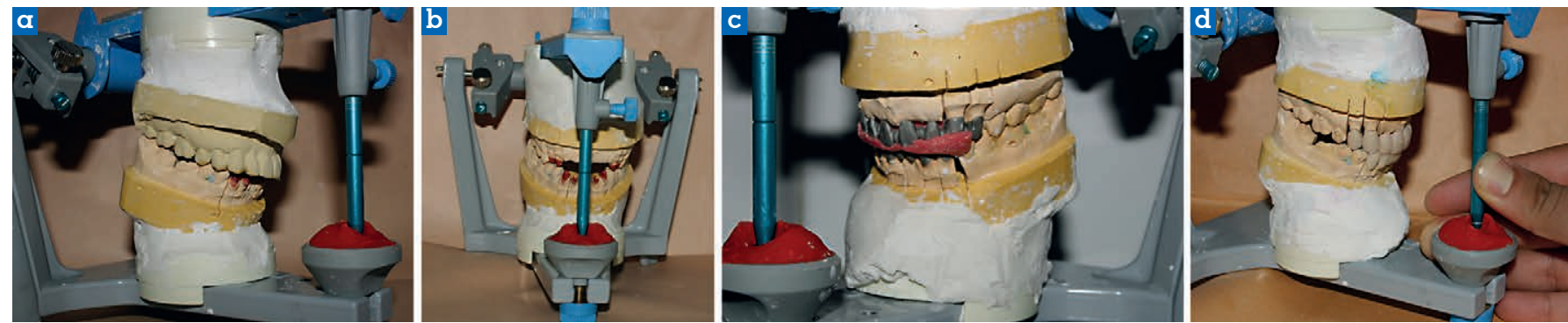

Fig. 14 :

a. Montage avec les modèles issus des provisoires

b. Montage avec le modèle supérieur de travail avec le modèle des prothèses provisoires inférieures

c. Montage avec le modèle inférieur de travail avec le modèle des prothèses provisoires supérieures

d. Montage avec les modèles de travail 


\section{Élaboration des prothèses définitives}

Le prothésiste procède à lélaboration des prothèses définitives en tenant compte bien sûr de la table incisive, aussi bien au stade des chapes qu'au stade de l'élaboration de la céramique (fig. 15).

Au stade de la céramique à létat de biscuit, locclusion dynamique est vérifiée en bouche (fig. 16).
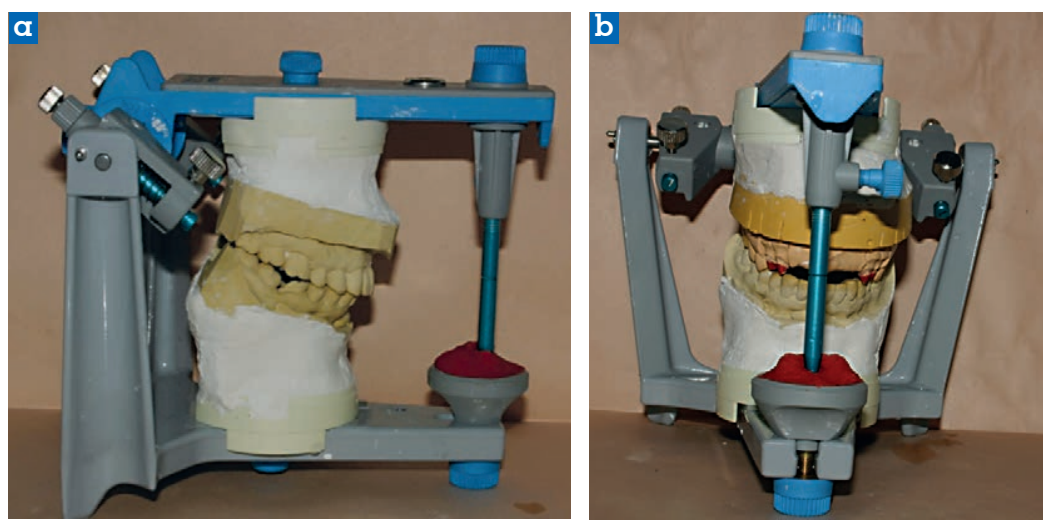

$\triangle$ Fig. 15 :

a. lélaboration des chapes métalliques en se référant à la table incisive programmée b. Le montage de la céramique en se référant à la table incisive programmée.
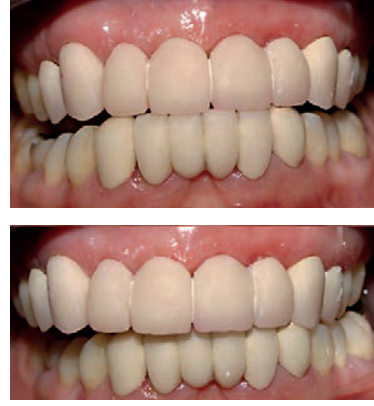

Fig. 16 :

Le résultat final occlusal et esthétique avec le sourire satisfait de la patiente.

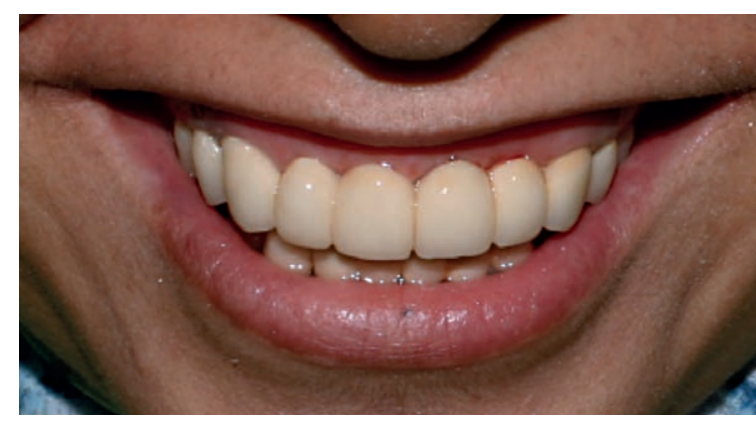

\section{CONCLUSION}

Dans les réhabilitations prothétiques antérieures, deux difficultés principales sont à retenir : la restauration de l'esthétique et du guide antérieur.

Les décisions thérapeutiques peuvent aller de limplantologie orale à la prothèse partielle adjointe, en passant par la prothèse fixée. Ces décisions ont toutes des exigences prothétiques, biologiques et biomécaniques. La chronologie du traitement est l'un des facteurs essentiels orientant la décision dans le but de faciliter le déroulement des différentes étapes thérapeutiques. Il faut organiser une analyse préprothétique rigoureuse afin détablir une stratégie thérapeutique tant au niveau des éventuelles phases chirurgicales parodontales que prothétiques.

\section{Bibliographie}

[1] Casteyde J-P. Locclusion de la canine. Importance, options de réglages, risques et précautions. AOS 2008;244:355-366.

[2] Chiche G, Pinault A. Esthétique et restauration des dents antérieures. Paris : Éditions CdP, 1995.

[3] Dawson PE. Les problèmes de l'occlusion clinique : évaluation, diagnostic, traitement. Paris : Éditions CdP, 1992
[4] Laplanche O, Pedeutour P, Laurent M, Mahler P, Orthlieb J-D. Le guide antérieur et ses anomalies : incidence sur la cinématique condylienne. Cah Prothèse 2002;117:43-55.

[5] Laurent M, Orthlieb J-D. Approche occlusale d'une restauration des incisives maxillaires. Cah Prothèse 1997; 99:11-19.

[6] Orthlieb J-D, El Zoghby A, Kordi M, Perez C. La fonction de guidage. Un modèle biomécanique pour un concept thérapeutique. Cah Prothèse 2004;128:43-53.
[7] Orthlieb J-D, Bezzina S, El Zoghby A, Giraudeau A. Reconstruction prothétique du guidage antérieur. Cah Prothèse 2004;128:55-64.

[8] Orthlieb J-D, Brocard D, Schittly J, Maniere-Ezvan A. Occlusodontie pratique. Paris : Éditions CdP, 2000.

[9] Orthlieb J-D. Gnathologie fonctionnelle. Vol 1 : Occlusion et restauration prothétique. Mémento. Paris : Éditions CdP, 2010.

[10] Valentin C., Yakhou O. Reconstitution du guide antérieur par des artifices de prothèse conjointe. Réalités cliniques 1993;4(2):163-176. 